\title{
Effects of respiratory motion on volumetric and positional difference of GTV in lung cancer based on 3DCT and 4DCT scanning
}

\author{
SHIJIANG WANG, DONGPING SHANG, XUE MENG, XINDONG SUN, YAN MA and JINMING YU \\ Department of Radiation Oncology, Shandong Cancer Hospital Affiliated to Shandong University, \\ Jinan, Shandong 250117, P.R. China
}

Received May 16, 2018; Accepted November 30, 2018

DOI: $10.3892 / \mathrm{ol} .2018 .9844$

\begin{abstract}
Differences in gross target volume (GTV) and central point positions among moving lung cancer models constructed by CT scanning at different frequencies were compared, in order to explore the effect of different respiratory frequencies on the GTV constructions in moving lung tumors. Eight models in different shapes and sizes were established to stimulate lung tumors. The three-dimensional computed tomography (3DCT) and four-dimensional computed tomography (4DCT) scanning were performed at 10,15 and 20 times/min in different models. Differences in GTV volumes and central point positions at different motion frequencies were compared by means of GTV3Ds $\left(\mathrm{GTV}_{3 \mathrm{D}-10}, \mathrm{GTV}_{3 \mathrm{D}-15}, \mathrm{GTV}_{3 \mathrm{D}-20}\right)$ and IGTV4Ds $\left(\mathrm{IGTV}_{4 \mathrm{D}-10}, \mathrm{IGTV}_{4 \mathrm{D}-15}, \mathrm{IGTV}_{4 \mathrm{D}-20}\right)$. Volumes of GTV ${ }_{3 \mathrm{D}-10}, \mathrm{GTV}_{3 \mathrm{D}-}$ ${ }_{15}, \mathrm{GTV}_{3 \mathrm{D}-20}$ were $12.41 \pm 14.26,10.38 \pm 11.18$ and $12.50 \pm 15.23 \mathrm{~cm}^{3}$ respectively $(\mathrm{P}=0.687)$. Central point coordinates in the $\mathrm{x}$-axis direction were $-8.16 \pm 96.21,-8.57 \pm 96.08$ and $-8.56 \pm 95.73$ respectively $(\mathrm{P}=0.968)$. Central point coordinates in the $y$-axis direction were $108.22 \pm 25.03,110.41 \pm 22.47$ and $109.04 \pm 24.24$ $(\mathrm{P}=0.028)$. Central point coordinates in the $\mathrm{z}$-axis direction were $65.19 \pm 13.68,65.43 \pm 13.40$ and $65.38 \pm 13.17(\mathrm{P}=0.902)$. The difference was significant in the $\mathrm{y}$-axis direction $(\mathrm{P}=0.028)$. Volumes of $\mathrm{IGTV}_{4 \mathrm{D}-10}, \mathrm{IGTV}_{4 \mathrm{D}-15}, \mathrm{IGTV}_{4 \mathrm{D}-20}$ were $17.78 \pm 19.42$, $17.43 \pm 19.56$ and $17.44 \pm 18.80 \mathrm{~cm}^{3}(\mathrm{P}=0.417)$. Central point coordinates in the $\mathrm{x}$-axis direction were $-7.73 \pm 95.93,-7.86 \pm 95.56$ and $-7.92 \pm 95.14(\mathrm{P}=0.325)$. Central point coordinates in the $\mathrm{y}$-axis direction were $109.41 \pm 24.54,109.60 \pm 24.13$ and $109.16 \pm 24.28$ $(\mathrm{P}=0.525)$. Central point coordinates in the $\mathrm{z}$-axis direction were $65.52 \pm 13.31,65.59 \pm 13.39$ and $65.51 \pm 13.34(\mathrm{P}=0.093)$. However, the central point position of GTV in the head and foot direction by 3DCT scanning was severely affected by the respiratory frequency.
\end{abstract}

Correspondence to: Dr Jinming Yu, Department of Radiation Oncology, Shandong Cancer Hospital Affiliated to Shandong University, 440 Jiyan Road, Jinan, Shandong 250117, P.R. China E-mail: yp83y5@163.com

Key words: respiratory frequency, gross tumor volume, central point position, 4DCT, 3DCT

\section{Introduction}

Radiation therapy is an important treatment for lung cancer. Radiotherapy simulation and target volumes are the key factors for curative effect of radiation therapy. In lung cancer radiotherapy, the patient's respiratory movement can cause changes in the target area, resulting in decreased treatment efficacy and increased adverse reactions, in order to give the target area accurate radiotherapy, we often give it a safe border in clinic (1). Respiratory movement is the main cause of lung tumor displacement and deformation, which is one of the uncertain factors in radiotherapy (2). Due to the influence of the environment, mental and physical states, the respiratory frequency may vary during the simulation and radiotherapy.

Tumor displacement and shape may change in the three-dimensional directions for the reason of physiological activities such as respiration. Research on respiratory gating and four-dimensional computed tomography (4DCT) has been done to reduce the influence of respiratory movement on the target volume $(3,4)$. The determination of internal target volume (ITV) based on 3DCT images in the free breathing state requires the expansion of 'safety margin' on the basis of gross target volume (GTV) $)_{3 \mathrm{D}}$. The margin is affected by many factors such as tumor location, tumor stage and lung volume (5). For example, the motion amplitude of the lower lobe tumor is larger than that of the upper lobe, and the motion amplitude in the head and foot direction is greater than that in the left and right direction. Also, the motion amplitude is smaller in the mediastinal and pleural tumors than that in the isolated lung tumors $(6,7)$.

3DCT can just capture the morphological and positional information instantaneously during the motion period. So, the 3DCT images contain limited motion information and cannot fully reflect the motion period of models. However, 4DCT can collect the motion information during the complete motion period. The reconstructed images can dynamically demonstrate the trajectory in the motion cycle, which can reflect the motion range of tumors in the three-dimensional directions (8).

Therefore, in order to further study and compare the characteristics of 3DCT and 4DCT scanning in the accurate radiotherapy of lung cancer, we designed eight models to 
Table I. Comparison of $\mathrm{GTV}_{3 \mathrm{D}}$ volumes and central point positions at three different motion frequencies.

\begin{tabular}{lcccc}
\hline Motion frequencies (times/min) & X-axis direction & y-axis direction & z-axis direction & Volumes $\left(\mathrm{cm}^{3}\right)$ \\
\hline 10 & $-8.16 \pm 96.21$ & $108.22 \pm 25.03$ & $65.19 \pm 13.68$ & $12.41 \pm 14.26$ \\
15 & $-8.57 \pm 96.08$ & $110.41 \pm 22.47$ & $65.43 \pm 13.40$ & $10.38 \pm 11.18$ \\
20 & $-8.56 \pm 95.73$ & $109.04 \pm 24.24$ & $65.38 \pm 13.17$ & $12.50 \pm 15.23$ \\
Z & 0.065 & 7.154 & 0.207 & 0.750 \\
P-value & 0.968 & 0.028 & 0.902 & 0.687 \\
\hline
\end{tabular}

GTV, gross target volume.

simulate lung tumors and analyze the effects of different respiratory frequencies on $3 \mathrm{D}$ target volume and 4D target volume.

\section{Materials and methods}

Equipment and parameter settings. We used Philips Big Bore CT scanner, Varian Real-time Position Management System, Modus breathing platform and 8 models in different shapes and sizes ( 3 spherical, 4 cuboid, and a cylinder one; volume range: $\left.0.52-33.50 \mathrm{~cm}^{3}\right)$. CT scanning condition was at $120 \mathrm{kV}, 300 \mathrm{mAs}$, with thickness of $3 \mathrm{~mm}$, the rotation period $1.5 \mathrm{sec}$, the detector combination conditions $16 \times 1.5 \mathrm{~mm}$, the screw pitch 0.938 , and the scanning bed forward speed of $22.5 \mathrm{~mm} / \mathrm{sec}$. The frequencies of the platforms were set at 10 , 15 and 20 times/min, respectively. The motion amplitude of lung in the three-dimensional directions was simulated by the models, which was set at $3 \mathrm{~mm}$ in the $\mathrm{x}$-axis, $12 \mathrm{~mm}$ in the $\mathrm{y}$-axis, and $3 \mathrm{~mm}$ in the z-axis, respectively $(9,10)$.

The study was approved by the Ethics Committee of Shandong Cancer Hospital Affiliated to Shandong University (Jinan, China). Signed informed consent was obtained from the patient or guardian.

CT scanning and image acquisition. The models were fixed on the breathing platform with an angle of $15^{\circ}$. The motion of the models was controlled by respiratory movement waveform of one patient at different respiratory frequencies [10, 15, 20 breathe per minute (BPM), respectively]. All models were scanned for 3DCT and 4DCT at three frequencies, respectively. Before 4DCT scanning, the infrared camera set at the end of the scanning bed was used to collect the motion trajectory of the models. Then, the motion signal of models was used to simulate the same patient's respiratory signal to the large aperture CT. The CT device started scanning after receiving the motion signal. Reconstruction of $\mathrm{CT}$ images in different phases $(0,10,20, \ldots, 90 \%)$ was carried out by $4 \mathrm{D}$ volumetric data.

Construction and definition of target volume. Tumor LOC software was used to construct $\mathrm{GTV}_{3 \mathrm{D}-10}, \mathrm{GTV}_{3 \mathrm{D}-15}$ and $\mathrm{GTV}_{3 \mathrm{D}-20}$ on 3DCT images at 10, 15, 20 times/min, respectively, by the same doctor, under the same window width and window level. Then the volumes and central point coordinates of GTV3Ds at three kinds of motion frequencies were obtained. GTVs on 4DCT images were delineated in ten phases at three motion frequencies as above. Then the GTVs fused together to get our
IGTVs. We defined them IGTV $\mathrm{ID}-10, \mathrm{IGTV}_{4 \mathrm{D}-15}$ and $\mathrm{IGTV}_{4 \mathrm{D}-20}$, and obtained their volumes and central point coordinates.

Statistical analysis. The SPSS17.0 (SPSS, Inc., Chicago, IL, USA) was used to perform Friedman test in order to analyse volumes and central point coordinates of $\mathrm{GTV}_{3 \mathrm{D}-10}$, $\mathrm{GTV}_{3 \mathrm{D}-15}$ and GTV $\mathrm{GD}_{20}$ at different motion frequencies. The same method was used to compare volumes and center point coordinates of IGTV $\mathrm{ID}-10, \mathrm{IGTV}_{4 \mathrm{D}-15}$ and $\mathrm{IGTV}_{4 \mathrm{D}-20}$ by $4 \mathrm{DCT}$ scanning. GTV $\mathrm{GD}_{3 \mathrm{D}}$ and $\mathrm{IGTV}_{4 \mathrm{D}}$ at the same motion frequency were also compared by Wilcoxon test. $\mathrm{P}<0.05$ was considered to indicate a statistically significant difference.

\section{Results}

Comparison of $G T V_{3 D}$ volumes and central point positions at three different motion frequencies. There was no significantly statistical difference among the volumes and the central point positions in the $\mathrm{x}$-axis and $\mathrm{z}$-axis directions at the three different motion frequencies, however, the central point coordinates in the $y$-axis direction were statistically different (Table I). The minimum $\mathrm{GTV}_{3 \mathrm{D}}$ central point coordinate values in the $\mathrm{x}$-axis direction were $-161.80,-161.50$ and -159.90 , and the maximum values were 102.17, 101.00 and 100.22 at three different motion frequencies, respectively. As for in the y-axis direction, the minimum coordinate values were $77.60,80.60$ and 77.60 and the maximum values were $145.10,142.10$ and 143.60 . In the z-axis direction, the minimum coordinate values were 39.45 , 40.23 and 41.02 , and the maximum values were $80.08,78.91$ and 79.30 .

Comparison of $I G T V_{4 D}$ volumes and central point positions at three different motion frequencies. There was no significant difference among the IGTV $\mathrm{V}_{4 \mathrm{D}}$ volumes and the central point positions at 10, 15 and 20 times/min (Table II). The minimum central point coordinate values of the IGTV $_{4 D}$ in the $x$-axis direction were $-161.10,-159.50$ and -158.11 , and the maximum values were $101.79,100.43$ and 99.40 , respectively. As for in the $\mathrm{y}$-axis direction, the minimum coordinate values were all 78.10, and the maximum values were $144.10,142.60$ and 143.60 , respectively. In the $\mathrm{z}$-axis direction, the minimum values were $40.44,40.33$ and 40.32 , and the maximum values were $79.30,79.45$ and 79.41 .

Comparison of $G T V_{3 D}$ and $I G T V_{4 D}$ volumes at the same motion frequency. The volumes of $\mathrm{IGTV}_{4 \mathrm{D}}$ were significantly larger 
Table II. Comparison of $\mathrm{IGTV}_{4 \mathrm{D}}$ volumes and central point positions at three different motion frequencies.

\begin{tabular}{lcccc}
\hline Motion frequencies (times/min) & x-axis direction & y-axis direction & z-axis direction & Volumes $\left(\mathrm{cm}^{3}\right)$ \\
\hline 10 & $-7.73 \pm 95.93$ & $109.41 \pm 24.54$ & $65.52 \pm 13.31$ & $17.78 \pm 19.42$ \\
15 & $-7.86 \pm 95.56$ & $109.60 \pm 24.13$ & $65.59 \pm 13.39$ & $17.43 \pm 19.56$ \\
20 & $-7.92 \pm 95.14$ & $109.16 \pm 24.28$ & $65.51 \pm 13.34$ & $17.44 \pm 18.80$ \\
Z & 2.250 & 1.273 & 4.750 & 1.750 \\
P-value & 0.325 & 0.529 & 0.093 & 0.417 \\
\hline
\end{tabular}

GTV, gross target volume; IGTV, internal gross tumor volume.

Table III. Comparison of $\mathrm{GTV}_{3 \mathrm{D}}$ and $\mathrm{IGTV}_{4 \mathrm{D}}$ volumes at the same motion frequency.

\begin{tabular}{lccc}
\hline Groups & 10 times/min & 15 times/min & 20 times/min \\
\hline GTV $_{\text {3D }}$ & $12.41 \pm 14.26$ & $10.38 \pm 11.18$ & $12.50 \pm 15.23$ \\
IGTV $_{\text {4D }}$ & $17.78 \pm 19.42$ & $17.43 \pm 19.56$ & $17.44 \pm 18.80$ \\
Z & -2.521 & -2.521 & -2.521 \\
P-value & 0.012 & 0.012 & 0.012 \\
\hline
\end{tabular}

GTV, gross target volume; IGTV, internal gross tumor volume.

than those of $\mathrm{GTV}_{3 \mathrm{D}}$ at three different motion frequencies, and the difference was statistically significant (Table III). The volume ratios of $\mathrm{IGTV}_{4 \mathrm{D}}$ and $\mathrm{GTV}_{3 \mathrm{D}}$ were $1.56 \pm 0.40,1.64 \pm 0.24$ and $1.61 \pm 0.53$, respectively. There was no significant difference in the volume ratio at different frequencies $(\mathrm{P}=0.687)$.

\section{Discussion}

At present, radiotherapy of lung cancer has entered the era of precise radiotherapy. The determination of target area is the key point of radiotherapy. The respiratory movement has a significant effect on the radiation dose distribution and radiotherapy effect of lung cancer, which is one of the important factors to be considered when the target region expands outside (11). Therefore, many scholars have carried out relevant research to reduce the influence of tumor displacement on the target area (12).

4DCT incorporates time factors into the 3D images and accurately reflects the changes of the motion range and spatial position of the chest tumor. While significantly reducing respiratory motion artifacts, the 4DCT accurately reflects the spatial movement and displacement of the target zone during the respiratory cycle information to reduce the surrounding normal tissue and organ exposure, target area leakage and target low-dose area caused by unification of border extension based on conventional CT localization images for ITV determination $(13,14)$.

CBCT online real-time monitoring correction technology of tumors and normal organs, which can adjust the treatment conditions according to changes in the position of the organs so that the irradiation field tightly 'follow' the target area, thereby reducing the setup error during radiotherapy (15).
In this study, we used models of different volumes to simulate lung tumor in order to analyse the impact of motion frequency on $\mathrm{GTV}_{3 \mathrm{D}}$. Results showed that there was no significant difference among the volumes of $\mathrm{GTV}_{3 \mathrm{D}}$ in the three different motion frequencies. The volume of $\mathrm{GTV}_{3 \mathrm{D}}$ was $11.52 \pm 13.37 \mathrm{~cm}^{3}$ when they were scanned in the static state. Moreover, there was no significant difference between the volumes of $\mathrm{GTV}_{3 \mathrm{D}}$ in the static state and in the three different motion states $(\mathrm{Z}=0.570$; $\mathrm{P}=0.903)$. The volumes of $\mathrm{GTV}_{3 \mathrm{D}}$ at 10 and 20 times/min were slightly larger than that in the static state, yet the volume of $\mathrm{GTV}_{3 \mathrm{D}}$ at 15 times/min was slightly smaller than that in the static state. The total number of GTV $\mathrm{VD}_{3 \mathrm{D}}$ volumes we constructed at the three motion frequencies was 24 . Among them, 12 cases were larger, 11 cases were smaller, and only 1 case was equal to the volume of $\mathrm{GTV}_{3 \mathrm{D}}$ in the static state. The GTV volume constructed by the 3DCT images in motion could be larger or smaller than that in the static state, and the form of the models would be stretched or compressed. The reason why there was difference in volumes was due to the presence of repeated scanning or omissive scanning (16). In the case of a spherical model, for example, when the motion direction of the model was just the same as that of the scanning bed in the y-axis direction, the velocity of the model was relatively slowed down if we regarded the bed as a reference. So, part of the model volume was repeatedly scanned and as a result, the volume of the $\mathrm{GTV}_{3 \mathrm{D}}$ constructed was larger than that in the static state. On the other hand, when the motion direction of the model was opposite to that of the scanning bed, the velocity of the model was relatively accelerated. So, part of the model volume would be omissive and as a result, the volume of the $\mathrm{GTV}_{3 \mathrm{D}}$ constructed is smaller than that in the static state. The repeated or omissive volumes of the scanned models were related to the direction and velocity of the scanning bed. When the model and the bed were in the same direction and the velocity was relatively slow, the repeated volume would increase and the form of the model would be stretched. On the other hand, when the model and the bed were in the opposite directions and the velocity was relatively quicker, the omissive volume increased and the form of the model was obviously compressed in the y-axis direction, then the volume of $\mathrm{GTV}_{3 \mathrm{D}}$ would significantly decrease.

There was no significant difference in the volumes of $\mathrm{GTV}_{3 \mathrm{D}}$ at three different frequencies, neither any significant difference in the central point positions in the $\mathrm{x}$-axis and $\mathrm{z}$-axis directions. However, there was significant difference in the $\mathrm{GTV}_{3 \mathrm{D}}$ central point positions in the $\mathrm{y}$-axis direction. Because of the quick CT scanning bed moving speed $(22.5 \mathrm{~mm} / \mathrm{sec}$, 
$16 \times 1.5 \mathrm{~mm}$ detector), the time for model scanning and CT images collection was very short. So the central point positions of $\mathrm{GTV}_{3 \mathrm{D}}$ at different motion frequencies were closely related to the positions of the models in the y-axis direction when they were scanned. Since the central point position of a model during the motion period was random in the y-axis direction, the larger motion amplitude in the y-axis direction and the limited motion information included in the 3DCT images were both the relevant factors which led to the difference of $\mathrm{GTV}_{3 \mathrm{D}}$ central point positions in the $\mathrm{y}$-axis direction.

The IGTV $_{4 \mathrm{D}}$ formed by GTVs in ten motion phases, contained all the information of the motion period. The planning target volume (PTV) based on the 4DCT scanning was superior to that based on 3DCT by the traditional axial scanning $(17,18)$. In the process of 4DCT scanning, the motion amplitudes of the models in the three-dimensional directions was the same at the three different motion frequencies. The difference of the IGTV volumes at the three motion frequencies was $<0.5 \mathrm{~cm}^{3}$, and the three-dimensional coordinates of the IGTV central points were not statistically different. That meant changes in frequencies could not change the motion information contained in the 4DCT volume scanning. The $\mathrm{GTV}_{3 \mathrm{D}}$ of the model constructed by 3D images was significantly smaller than that constructed by 4DCT images at the same motion frequency. The difference was statistically significant. The volume ratios of $\mathrm{IGTV}_{4 \mathrm{D}} / \mathrm{GTV}_{3 \mathrm{D}}$ were $1.56 \pm 0.40,1.64 \pm 0.24$ and $1.61 \pm 0.53$ at 10 , 15 and 20 times/min, respectively. The same amplitude was the key reason why IGTV $\mathrm{ID}_{\mathrm{D}} / \mathrm{GTV}_{3 \mathrm{D}}$ volume ratios were close to each other at different frequencies. The motion information collected by 3DCT is very limited, and the safety margin is based on experience (19). It may cause omission of tumor or excessive exposure of normal tissues in radiotherapy, and the volume of omission can reach as much as $25 \%$ (20). IGTV constructed by 4DCT not only contains complete motion information, but also decreases the image artifacts. In this way, the image quality has significantly improved. So 4DCT is currently the best method to get chest tumor motion information caused by respiration and to construct individual target volumes $(21,22)$.

In conclusion, under the condition of the same motion amplitude, change of motion frequency has little effect on the construction of 4DCT target volume, the size of 3DCT target volume and the central point position not only in the left and right direction but also in the forward and backward direction. The position in the head and foot direction is obviously affected, and $\mathrm{GTV}_{3 \mathrm{D}}$ constructed in different motion periods have great deformation.

\section{Acknowledgements}

Not applicable.

\section{Funding}

This project is financially supported by Shandong Provincial Key Program of Research and Development (2016CYJS01A03).

\section{Availability of data and materials}

The datasets used and/or analyzed during the present study are available from the corresponding author on reasonable request.

\section{Authors' contributions}

SW and DS drafted this manuscript and helped with CT scanning. XM and XS contributed to the construction and definition of target volume. YM and JY helped with statistical analysis. All authors read and approved the final manuscript.

\section{Ethics approval and consent to participate}

The study was approved by the Ethics Committee of Shandong Cancer Hospital Affiliated to Shandong University (Jinan, China). Signed informed consent was obtained from the patient or guardian.

\section{Patient consent for publication}

Not applicable.

\section{Competing interests}

The authors declare that they have no competing interests.

\section{References}

1. Jang SS, Huh GJ, Park SY, Yang PS, Chung HN, Seo JH, Park JC, Yang YJ and Cho EY: Reconstitution of internal target volumes by combining four-dimensional computed tomography and a modified slow computed tomography scan in stereotactic body radiotherapy planning for lung cancer. Radiat Oncol 9: 106, 2014.

2. Ahmed N, Venkataraman S, Johnson K, Sutherland K and Loewen SK: Does motion assessment with 4-dimensional computed tomographic imaging for non-small cell lung cancer radiotherapy improve target volume coverage? Clin Med Insights Oncol 11: 1179554917698461, 2017.

3. Gagel B, Demirel C, Kientopf A, Pinkawa M, Piroth M, Stanzel S, Breuer C, Asadpour B, Jansen T, Holy R, et al: Active breathing control $(\mathrm{ABC})$ : Determination and reduction of breathing-induced organ motion in the chest. Int $\mathrm{J}$ Radiat Oncol Biol Phys 67: 742-749, 2007.

4. Sarudis S, Karlsson Hauer A, Nyman J and Bäck A: Systematic evaluation of lung tumor motion using four-dimensional computed tomography. Acta Oncol 56: 525-530, 2017.

5. Jang SS, Huh GJ, Park SY, Yang PS and Cho E: Usefulness of target delineation based on the two extreme phases of a four-dimensional computed tomography scan in stereotactic body radiation therapy for lung cancer. Thorac Cancer 6: 239-246, 2015.

6. Knybel L, Cvek J, Molenda L, Stieberova N and Feltl D: Analysis of lung tumor motion in a large sample: Patterns and factors influencing precise delineation of internal target volume. Int $\mathrm{J}$ Radiat Oncol Biol Phys 96: 751-758, 2016.

7. Jan N, Hugo GD, Mukhopadhyay N and Weiss E: Respiratory motion variability of primary tumors and lymph nodes during radiotherapy of locally advanced non-small-cell lung cancers. Med Phys 41: 021718, 2014.

8. Ono T, Nakamura M, Hirose Y, Kitsuda K, Ono Y, Ishigaki T and Hiraoka $\mathrm{M}$ : Estimation of lung tumor position from multiple anatomical features on 4D-CT using multiple regression analysis. J Appl Clin Med Phys 18: 36-42, 2017.

9. Hof H, Rhein B, Haering P, Kopp-Schneider A, Debus J and Herfarth K: 4D-CT-based target volume definition in stereotactic radiotherapy of lung tumors: Comparison with a conventional technique using individual margins. Radiother Oncol 93: 419-423, 2009.

10. Weiss E, Wijesooriya K, Dill SV and Keall PJ: Tumor and normal tissue motion in the thorax during respiration: Analysis of volumetric and positional variations using 4D CT. Int J Radiat Oncol Biol Phys 67: 296-307, 2007.

11. Lee SY,Lim S, Ma SY and Yu J: Gross tumor volume dependency on phase sorting methods of four-dimensional computed tomography images for lung cancer. Radiat Oncol J 35: 274-280, 2017. 
12. Inata $\mathrm{H}$, Araki F, Kuribayashi $\mathrm{Y}$, Hamamoto $\mathrm{Y}$, Nakayama $\mathrm{S}$, Sodeoka N, Kiriyama T and Nishizaki O: Development of a real-time monitoring system for intra-fractional motion in intracranial treatment using pressure sensors. Phys Med Biol 60: 7229-7243, 2015.

13. Patel AA, Wolfgang JA, Niemierko A, Hong TS, Yock T and Choi NC: Implications of respiratory motion as measured by four-dimensional computed tomography for radiation treatment planning of esophageal cancer. Int J Radiat Oncol Biol Phys 74: 290-296, 2009.

14. Fleckenstein J, Kremp K, Kremp S, Palm J and Rübe C: IMRT and $3 \mathrm{D}$ conformal radiotherapy with or without elective nodal irradiation in locally advanced NSCLC: A direct comparison of PET-based treatment planning. Strahlenther Onkol 192: 75-82, 2016.

15. Shimohigashi Y, Toya R, Saito T, Ikeda O, Maruyama M, Yonemura K, Nakaguchi Y, Kai Y, Yamashita Y, Oya N, et al: Tumor motion changes in stereotactic body radiotherapy for liver tumors: An evaluation based on four-dimensional cone-beam computed tomography and fiducial markers. Radiat Oncol 12: 61, 2017.

16. Pan T, Martin RM and Luo D: New prospective 4D-CT for mitigating the effects of irregular respiratory motion. Phys Med Biol 62: N350-N361, 2017.

17. Rietzel E, Chen GT, Choi NC and Willet CG: Four-dimensional image-based treatment planning: Target volume segmentation and dose calculation in the presence of respiratory motion. Int J Radiat Oncol Biol Phys 61: 1535-1550, 2005.
18. Wang N, Patyal B, Ghebremedhin A and Bush D: Evaluation and comparison of New 4DCT based strategies for proton treatment planning for lung tumors. Radiat Oncol 8: 73, 2013.

19. Li F, Li J, Zhang Y, Xu M, Shang D, Fan T, Liu T and Shao Q: Geometrical differences in gross target volumes between 3DCT and 4DCT imaging in radiotherapy for non-small-cell lung cancer. J Radiat Res (Tokyo) 54: 950-956, 2013.

20. Kamomae T, Monzen H, Nakayama S, Mizote R, Oonishi Y, Kaneshige $\mathrm{S}$ and Sakamoto T: Accuracy of image guidance using free-breathing cone-beam computed tomography for stereotactic lung radiotherapy. PLoS One 10: e0126152, 2015.

21. Bai T, Zhu J, Yin Y, Lu J, Shu H, Wang L and Yang B: How does four-dimensional computed tomography spare normal tissues in non-small cell lung cancer radiotherapy by defining internal target volume? Thorac Cancer 5: 537-542, 2014.

22. Vedam SS, Keall PJ, Kini VR, Mostafavi H, Shukla HP and Mohan R: Acquiring a four-dimensional computed tomography dataset using an external respiratory signal. Phys Med Biol 48: 45-62, 2003.

This work is licensed under a Creative Commons Attribution-NonCommercial-NoDerivatives 4.0 International (CC BY-NC-ND 4.0) License. 